\title{
Distância fenotípica entre matrizes de açaizeiro (Euterpe oleracea Mart.) procedentes do nordeste do Pará
}

\author{
Phenotypic distance among assai palm's mother plants \\ (Euterpe oleracea Mart.) from Eastern Amazon
}

\author{
Rosemiro dos Santos Galate ${ }^{1 *}$; Milton Guilherme da Costa Mota ${ }^{2}$; \\ José Maria Demetrio Gaia ${ }^{3}$; Merilene do Socorro Silva Costa ${ }^{4}$
}

\begin{abstract}
Resumo
O açaizeiro (Euterpe oleracea Mart.) é uma palmácea nativa do Estuário Amazônico. Adivulgação de suas propriedades nutracêuticas, sua entrada nos mercados nacional e internacional e o consequente aumento de sua área plantada na Amazônia, fazem com que haja necessidade de estudar seu cultivo racional, já que o extrativismo e o manejo de açaizais nativos apresentam produção limitada. O objetivo foi realizar uma estimativa preliminar da variabilidade e parâmetros genéticos e avaliar a divergência fenotípica de matrizes de açaizeiro. Tomaram-se dados de 129 matrizes de açaizeiro do Nordeste Paraense. Estimouse, com base em 22 caracteres morfoagronômicos relacionados ao desenvolvimento e produção da planta, a ANOVA e parâmetros genéticos. A divergência foi estimada pela distância euclidiana média padronizada com os métodos de agrupamento UPGMA e de Tocher. A ANOVA apresentou $77,3 \%$ dos caracteres com variância significativa ( $\mathrm{p} \leq 0,01$ e $\mathrm{p} \leq 0,05)$; a razão $\mathrm{CVg} / \mathrm{CVe}$ indicou que $72,3 \%$ dos caracteres podem apresentar variabilidade genética para utilização em programas de melhoramento. A distância euclidiana detectou como menos divergentes as matrizes EO-070 e EO-072, ambas da Ilha do Combu e as mais divergentes foram EO-010 (Belém) e EO-018 (Salinópolis). Os métodos de agrupamento formaram dez (Tocher) e nove (UPGMA) grupos, praticamente semelhantes. Pelo método de Tocher as matrizes mais dissimilares foram EO-035 (Capitão Poço), EO-109 (Ilha do Combu), EO019 (Salinópolis) e EO-010 (Belém); e pelo método UPGMA EO-010 (Belém), EO-011 (São João de Pirabas), EO-017, EO-018 e EO-019 (Salinópolis); EO-062 e EO-109 (Ilha do Combu). Concluiu-se que as 129 matrizes apresentam elevada variabilidade fenotípica. Os parâmetros genéticos indicaram que há possibilidade de utilização dessa variabilidade em programas de melhoramento genético. Há matrizes divergentes para utilização como genitores na obtenção de genótipos superiores.
\end{abstract}

Palavras-chave: Euterpe oleracea Mart., características morfológicas, variabilidade genética, divergência genética

\begin{abstract}
The açai palm tree (Euterpe oleracea Mart.) is native to the Amazonian estuary. Media revelation of the nutraceutical properties of açai palm has resulted in its introduction in the national and international markets, consequently increasing the açai palm crop area in the Amazon. Nevertheless, açai palm cultivation is performed in a rational manner, because of limited product extraction from the native açai palm varieties. The study aimed to conduct a preliminary estimate of genetic variability, parameters,
\end{abstract}

\footnotetext{
${ }^{1}$ Eng $^{\mathrm{o}}$ Agr $^{\mathrm{o}}$, Discente de Doutorado em Agroecossistemas da Amazônia e Mestre da Universidade Federal Rural da Amazônia, Instituto Ciberespacial, UFRA/ICIBE, Belém, PA. E-mail: rosemiro.galate@ufra.edu.br

${ }^{2}$ Eng $^{\circ}$ Agro ${ }^{\circ}$, M.e Prof. Visitante da UFRA/ICIBE, Belém, PA. E-mail: mota@amazon.com.br

${ }^{3}$ Eng $^{\circ}$ Agr $^{\circ}$, M.e da UFRA/ICIBE, Belém, PA. Bolsista DCR, CNPq-FAPESPA. E-mail: josegaia@globo.com

${ }^{4}$ Eng $^{\text {a }}$ Florestal, M.e Prof ${ }^{\text {a }}$ da UFRA/ICIBE, Belém, PA. E-mail: merilene@hotmail.com

* Autor para correspondência
} 
and phenotypic divergence of parent açai palms. Data from 129 parent plants from northeast Pará was collected. Analysis of variance (ANOVA) of 22 morphoagronomic traits was performed to estimate the genetic parameters. Divergence was estimated using standardized average euclidean distance by using unweighted pair group method with arithmetic mean (UPGMA) and Tocher clustering methods. Significant variance was noted in $77.3 \%$ traits (ANOVA, $\mathrm{p}<0.01$ and $\mathrm{p}<0.05$ ); $\mathrm{CVg} / \mathrm{CVe}$ ratio indicated that $72.3 \%$ traits may present genetic variability for use in breeding programs. The euclidean distance showed EO-070 and EO-072 parent plants (Combu Island) as the least divergent, and EO-010 (Belém) and EO-018 (Salinópolis) as the most divergent. The clustering methods determined ten (Tocher) and nine (UPGMA) similar groups. The most dissimilar parent plants were EO-035 (Capitão Poço), EO109 (Combu Island), EO-019 (Salinópolis), and EO-010 (Belém) (Tocher method), whereas even the EO-010 (Belém); EO-011 (São João de Pirabas); EO-017, EO-018, and EO-019 (Salinópolis); EO-062 and EO-109 (Combu Island) plants showed dissimilarity. We concluded that the 129 mother plants have high phenotypic variability, indicating the possibility of their use in genetic breeding programs. Further, the divergent parent plants can be used in the production of genotypes with favorable characteristics.

Key words: Euterpe oleracea Mart., morphologic traits, genetic variability, genetic divergence

\section{Introdução}

$\mathrm{O}$ açaizeiro (Euterpe oleracea Mart.) é uma palmácea nativa do Estuário Amazônico, que habita áreas permanente ou temporariamente inundáveis. Os produtos derivados do extrativismo do açaizeiro - fruto e palmito - ocupam lugar de destaque na economia do Estado do Pará e os frutos são de fundamental importância para a subsistência de populações ribeirinhas, pelo alimento que proporcionam e pela comercialização do excedente familiar (HOMMA, 2006).

O aumento da área plantada com a cultura na Amazônia, em função de sua entrada nos mercados nacional e internacional suscita seu cultivo racional, já que o extrativismo e o manejo de açaizais nativos não serão capazes de atender a crescente demanda, pois apresentam baixa produção, baixo rendimento de frutos e de polpa, além de irregularidade na maturação dos frutos, variação na tonalidade violácea dos frutos maduros, plantas com caule solitário ou com variações no perfilhamento, baixa tolerância ao déficit hídrico, dentre outros (OLIVEIRA; FERREIRA; SANTOS, 2007; SANTANA; CARVALHO; MENDES, 2008).

Estudos importantes sobre caracterização de germoplasma de açaizeiro sobre a coleção de germoplasma da Embrapa Amazônia Oriental foram desenvolvidos por Oliveira, Ferreira e
Santos (2006), ao estudar a seleção de descritores fenotípicos por análise de componentes principais (ACP) associada a critérios de descarte de caracteres contendo menor contribuição para a variabilidade, assim como a divergência genética entre acessos de açaizeiro utilizando descritores morfoagronômicos (OLIVEIRA; FERREIRA; SANTOS, 2007); além da avaliação genética de progênies de polinização aberta com estimativa de parâmetros genéticos (FARIAS NETO et al., 2007). Yokomizo et al. (2010) também avaliaram progênies de açaizeiro. Em outras espécies de palmeiras, Manfio (2010) estimou parâmetros genéticos de progênies e divergência genética em matrizes de macaúba (Acrocomia aculeata); Farias Neto e Oliveira (2012) avaliaram a distância genética em acessos de tucumanzeiro (Astrocaryum vulgare Mart.).

O exame dos dados morfológicos é importante para a caracterização e a avaliação morfoagronômica de germoplasma. A análise da distância genética é uma ferramenta auxiliar de grande importância em programas de melhoramento. Sua estimativa pode informar a respeito da organização do germoplasma e auxiliar na definição de cruzamentos artificiais. A seleção de genitores dissimilares permite a obtenção de populações segregantes com ampla variabilidade genética e elevada frequência de indivíduos transgressivos, já que a heterose e a capacidade específica de combinação entre dois genitores 
dependem da existência de dominância no controle do caráter e da presença de diferenças genéticas entre os genótipos (FALCONER; MACKAY, 1996; VIEIRA et al., 2007).

Este trabalho teve como objetivo realizar uma avaliação preliminar da variabilidade fenotípica e genética, e avaliar a divergência genética com base em caracteres morfoagronômicos de matrizes de açaizeiro amostradas no nordeste paraense, visando selecionar indivíduos divergentes para futuros cruzamentos.

\section{Material e Métodos}

Foram coletados dados de 129 matrizes de açaizeiro amostradas em ambiente antropizados de terra firme e de várzea, procedentes de Belém (cidade, Ilha do Combu e Ilha do Mosqueiro) e de seis municípios do Nordeste Paraense, de diferentes climas classificados de acordo com Koppen (Tabela 1). A coleção estudada é, em sua maior parte, procedente da Ilha do Combu, devido ao fato de fornecer a maior proporção do açaí consumido em Belém-PA.

Tabela 1. Caracterização de 129 matrizes de açaizeiro quanto à microrregião, local, ponto de coleta, posicionamento global e ao clima, amostradas no Nordeste Paraense. Belém-PA, UFRA, 2012.

\begin{tabular}{cccc}
\hline Matrizes & Número de matrizes & Local & Clima \\
\hline EO-001 & 1 & Belém & Afi \\
EO-002 a EO-007 & 6 & Ilha de Mosqueiro & Afi \\
EO-008 a EO-010 & 3 & Belém & Afi \\
EO-011 a EO-014 & 4 & São João de Pirabas & Ami \\
EO-015 a EO-021 & 7 & Salinópolis & Ami \\
EO-022 a EO-028 & 7 & Santarém Novo & Ami \\
EO-029 a EO-032 & 4 & São João de Pirabas & Ami \\
EO-033 a EO-040 & 8 & Capitão Poço & Ami \\
EO-041 a EO-043 & 3 & Ourém & Ami \\
EO-044 a EO-053 & 10 & Capitão Poço & Ami \\
EO-054 a EO-094 & 41 & Ilha do Combu & Afi \\
EO-095 a EO-107 & 13 & Ilha do Combu & Afi \\
EO-108 a EO-129 & 22 & Ilha do Combu & Afi \\
\hline
\end{tabular}

Fonte: Elaboração dos autores.

Analisaram-se 22 caracteres morfométricos relativos à planta e aos frutos. Os caracteres da planta foram: (1) Número de estipe por planta (NEP); (2) Altura do estipe mais velho da planta (AE, m); (3) Número de folhas existentes no estipe mais velho da planta (NF); (4) Circunferência do estipe mais velho da planta à altura do peito $(\mathrm{CAP}, \mathrm{cm}) \mathrm{e}$; (5) Comprimento do entrenó $(\mathrm{CEN}, \mathrm{cm})$, referente à distância entre os nós (cicatrizes foliares) do estipe mais velho da planta, tendo como ponto de partida a altura do peito para baixo.

Os caracteres dos frutos foram avaliados em apenas um cacho por genótipo, sendo retirados, aleatoriamente, dez frutos. Mediu-se : (6) Diâmetro longitudinal do fruto (DLF, mm); (7) Diâmetro transversal do fruto (DTF, mm); (8) Massa do fruto (PF, g); (9) Massa da semente (PS, g); (10) Massa da polpa (PP, g), calculado pela diferença entre os caracteres PF e PS; (11) Rendimento de polpa por fruto (RPF, \%).

Os caracteres agronômicos foram: (12) Massa do cacho (PC, kg); (13) Massa de frutos por cacho (PFC, kg); (14) Rendimento de frutos por cacho (RFC, \%); (15) Número de ráquilas por cacho (NRC); (16) Número de frutos por ráquila (NFR); (17) Número de frutos por cacho (NFC); (18) 
Número de cachos por planta (NCP); (19) Massa de cem frutos (PCF, g) (20) Circunferência do cacho (CIC, cm); (21) Comprimento total do cacho (CC, $\mathrm{cm})$ e ; (22) Produção total de frutos (PTF, kg).

Para apreciação dos caracteres, utilizaram-se as análises de variância univariada (ANOVA) e multivariada de agrupamento, sendo a ANOVA utilizada apenas para verificar a variância das procedências e compará-las com outras citadas em literatura; para isso, considerou-se o modelo inteiramente casualizado, em cujos tratamentos foram consideradas 10 procedências, sendo a Ilha do Combu subdividida em três procedências. Parâmetros genéticos foram estimados para servir como um indicativo da variabilidade genética dessas matrizes.

Para viabilizar a análise de agrupamento, utilizou-se, como medida de dissimilaridade a distância euclidiana padronizada média entre duas plantas (matrizes) $X_{i k}$ e $X_{j k}, i \neq j$. É uma medida de dissimilaridade definida como a raiz quadrada da soma dos quadrados das diferenças entre os pares de matrizes (i e j) sobre p caracteres, correspondente à expressão matemática $\left(\mathrm{X}_{\mathrm{i}}, \mathrm{X}_{\mathrm{j}}\right)=\sqrt{\frac{1}{p} \sum_{k=1}^{p}\left(\mathrm{X}_{\mathrm{ik}}-\mathrm{X}_{\mathrm{jk}}\right)^{2}}$, em que $X_{i k}$ é o valor do caráter $k$ referente à matriz $\mathrm{i}$ e $\mathrm{X}_{\mathrm{jk}}$ representa o mesmo caráter k para a matriz j. Os dados foram padronizados pelo método do desvio padrão 1 .

Para análise de agrupamento, a variância dos caracteres foi previamente otimizada por componentes principais, sendo selecionados 14 dos 22 caracteres originais, a saber, NEP, AE, NF, CAP, CEN, DLF, PS, PP, PC, RFC, NRC, CC, CIC e NCP (GALATE et al., 2012) para realização dos agrupamentos. Os métodos de agrupamento utilizados foram o hierárquico UPGMA e o não hierárquico de otimização de Tocher. O Método UPGMA foi utilizado na delimitação dos grupos e para estabelecer um dendrograma para identificar grupos de matrizes, sendo a distância entre eles representada por $\mathrm{d}_{(\mathrm{i} j) \mathrm{k}}=$ média $\left\{\mathrm{d}_{\mathrm{i} k}, \mathrm{~d}_{\mathrm{jk}}\right\}$. No método de Tocher, os grupos são estabelecidos por meio da distância entre o indivíduo $\mathrm{k}$ e o grupo formado pelos indivíduos $\mathrm{ij}$, dada por $\mathrm{d}_{(\mathrm{ij}) \mathrm{k}}=\mathrm{d}_{\mathrm{ik}}+\mathrm{d}_{\mathrm{ij}}$, em que a entrada, ou não, do indivíduo k no grupo é feita considerando-se que se

$\frac{\mathrm{d}_{\text {(grupo)k }}}{\mathrm{n}} \leq \theta$,

inclui-se o indivíduo k no grupo e; se $\frac{\mathrm{d}_{\text {(grupo)k }}}{\mathrm{n}}>\theta$,

o indivíduo k não é incluído no grupo; sendo n é o número de indivíduos que constitui o grupo original. Os cálculos foram executados pela utilização do programa GENES (CRUZ, 2006, 2008).

\section{Resultados e Discussão}

Os resultados obtidos na análise da variância, com base nos 22 caracteres avaliados, apresentaram variabilidade entre as 129 matrizes, pelo teste $\mathrm{F}(\mathrm{p} \leq 0,01$ e $\mathrm{p} \leq 0,05)$ de $77,27 \%$ dos caracteres, indicando a existência de variação genética, possibilitando a identificação de genótipos promissores e obtenção de ganhos por seleção; entretanto o teste não indicou diferença significativa para os caracteres comprimento do entrenó, peso do cacho, comprimento do cacho, circunferência do cacho e número de cachos por planta (Tabela 2).

Ao comparar-se tais resultados com os de Oliveira, Ferreira e Santos (2007) que, analisando 87 acessos com base em 28 caracteres de açaizeiro procedentes dos Estados do Maranhão (9), Amapá (10) e Pará (68) constataram diferenças significativas entre os acessos, pelo teste $\mathrm{F}(\mathrm{p} \leq 0,01$ e $\mathrm{p} \leq 0,05)$ para 26 , correspondente à $92,86 \%$ dos descritores examinados, com exceção da duração da fase masculina e rendimento de polpa por fruto, verificou-se que a variabilidade detectada por estes autores foi proporcionalmente maior, possivelmente pela maior diversidade de procedências, oriundas de três Estados Federativos, sendo que nas procedências do Estado do Pará, apenas a Ilha de Mosqueiro foi comum aos dois estudos. 
Tabela 2. Estimativas da análise da variância obtidas para 22 caracteres morfoagronômicos avaliados em 129 matrizes de açaizeiro em seis municípios do Nordeste Paraense. Belém-PA, UFRA, 2012.

\begin{tabular}{|c|c|c|c|c|c|}
\hline Caráter & QMT & & QMR & $\mathbf{F}$ & $\mathbf{P}$ \\
\hline NEP & 0,993 & $*$ & 0,456 & 2,177 & 0,028 \\
\hline $\mathbf{A E}$ & 16,603 & $* *$ & 2,261 & 7,344 & 0,000 \\
\hline NF & 0,073 & $* *$ & 0,029 & 2,527 & 0,008 \\
\hline CAP & 110,626 & $* *$ & 33,4 & 3,312 & 0,001 \\
\hline CEN & 18,577 & ns & 15,415 & 1,205 & 0,298 \\
\hline DTF & 3,562 & $* *$ & 0,525 & 6,789 & 0,000 \\
\hline DLF & 6,075 & $* *$ & 0,775 & 7,839 & 0,000 \\
\hline PF & 0,071 & $* *$ & 0,022 & 3,164 & 0,002 \\
\hline PS & 0,079 & $* *$ & 0,016 & 4,904 & 0,000 \\
\hline PP & 0,01 & $* *$ & 0,004 & 2,414 & 0,015 \\
\hline RPF & 30,196 & $* *$ & 7,103 & 4,252 & 0,000 \\
\hline PC & 1,892 & $\mathrm{~ns}$ & 1,232 & 1,535 & 0,143 \\
\hline PFC & 2,603 & $*$ & 1,201 & 2,167 & 0,029 \\
\hline RFC & 167,553 & $* *$ & 41,206 & 4,066 & 0,000 \\
\hline NRC & 1,616 & $* *$ & 0,583 & 2,769 & 0,006 \\
\hline NFR & 2,067 & $* *$ & 0,599 & 3,452 & 0,001 \\
\hline $\mathrm{CC}$ & 221,502 & ns & 171,663 & 1,29 & 0,249 \\
\hline CIC & 516,378 & ns & 345,036 & 1,497 & 0,157 \\
\hline PCF & 834,514 & $* *$ & 275,656 & 3,027 & 0,003 \\
\hline NCP & 0,256 & ns & 0,196 & 1,304 & 0,242 \\
\hline NFC & 264,474 & $* *$ & 68,91 & 3,838 & 0,000 \\
\hline PTF & 117,429 & $* *$ & 40,305 & 2,913 & 0,004 \\
\hline
\end{tabular}

Fonte: Elaboração dos autores.

As estimativas da variância genotípica de 77,27 $\%$ dos caracteres foram superiores em relação à variância ambiental e os valores do coeficiente de variação relativa $(\mathrm{CVg} / \mathrm{CVe})$ maiores que a unidade, exceto para os caracteres que não apresentaram variabilidade significativa (Tabelas 3, 4 e 5). A razão $\mathrm{CVg} / \mathrm{CVe}$ determina um parâmetro genético útil para selecionar genótipos, quando este parâmetro atinge o valor igual ou superior a um, recomendando ao melhorista, que há possibilidade de fazer seleção entre populações, indicando que o caráter pode ser trabalhado no melhoramento (VENCOVSKY; BARRIGA, 1992). Suas estimativas variaram de 0,46, para o caráter comprimento do entrenó, a 2,56, para diâmetro transversal do fruto (Tabela 3).

Manfio (2010), ao estudar 51 progênies oriundas de matrizes de macaúba (Acrocomia aculeata) procedentes de Minas Gerais e São Paulo, verificou, pelos mesmos parâmetros, que houve variabilidade genética, selecionando 25 progênies. Farias Neto et al. (2007), ao obter parâmetros genéticos de 50 progênies de açaizeiro pelo método REM/BLUP, em látice 5x5, no Estado do Pará (Tomé-Açu), para cinco caracteres verificou que apenas três caracteres, altura, número de folhas e comprimento dos entrenós, apresentaram variação genética expressiva. Por outro lado, Yokomizo et al. (2010), na avaliação de 100 progênies de açaizeiro, procedentes do leste da Ilha do Marajó, por meio de 10 caracteres agronômicos do estipe, folha e cacho, no Estado do Amapá (Mazagão), em blocos ao acaso com duas repetições, encontrou baixos valores para a razão $\mathrm{CVg} / \mathrm{CVe}$ (inferiores a 0,80 ) e herdabilidade (inferiores a 52\%) para os caracteres avaliados, havendo, portanto, certa discordância com o Trabalho anterior, talvez, em função do uso de metodologias diferentes, apesar da utilização de procedências similares. 
Tabela 3. Estimativas de parâmetros genéticos obtidos da análise de 129 matrizes de açaizeiro coletadas em seis municípios do Nordeste Paraense em relação aos caracteres NEP, AE, NF, CAP, CEN, DTF e DLF. Belém, UFRA, 2012.

\begin{tabular}{lrrrrrrr}
\hline \multicolumn{1}{c}{ Parâmetros } & NEP & AE(m) & NF & CAP(cm) & CEN(cm) & DTF(mm) & DLF(mm) \\
Vf & 0,09 & 1,40 & 0,003 & 9,34 & 1,57 & 0,30 & 0,52 \\
Ve & 0,05 & 0,19 & 0,001 & 2,82 & 1,30 & 0,04 & 0,07 \\
Vg & 0,04 & 1,21 & 0,002 & 6,52 & 0,27 & 0,26 & 0,45 \\
CVf (\%) & 10,21 & 14,90 & 1,64 & 7,93 & 8,01 & 4,14 & 6,32 \\
CVe (\%) & 7,22 & 5,49 & 0,95 & 4,36 & 7,29 & 1,51 & 2,34 \\
CVg (\%) & 8,07 & 13,85 & 1,34 & 6,63 & 3,32 & 3,86 & 5,94 \\
CVr & 0,89 & 2,52 & 1,41 & 1,52 & 0,46 & 2,56 & 2,54 \\
MÉDIA & 2,77 & 7,94 & 3,34 & 38,50 & 15,64 & 13,22 & 11,30 \\
\hline
\end{tabular}

NEP: número de estipe por planta; AE: altura do estipe; NF: número de folhas; CAP: circunferência do estipe; CEN: comprimento do entrenó; DTF: diâmetro transversal do fruto; DLF: diâmetro longitudinal do fruto; Vf: variância fenotípica; Ve: variância ambiental; Vg: variância genética; CVf: coeficiente de variação fenotípica; CVe: coeficiente de variação ambiental; CVg: coeficiente de variação genética; $\mathrm{CVr}=\mathrm{CVg} / \mathrm{CVe}$ : coeficiente de variação relativa.

Fonte: Elaboração dos autores.

Tabela 4. Estimativas de parâmetros genéticos obtidos da análise de 129 matrizes de açaizeiro coletadas em seis municípios do Nordeste Paraense em relação aos caracteres PF, PS, PP, RPF, PC, PFC e RFC. Belém-PA, UFRA, 2012.

\begin{tabular}{lrrrrrrr}
\hline \multicolumn{1}{c}{ Parâmetros } & PF(g) & PS(g) & PP(g) & RPF(\%) & PC(kg) & PFC(kg) & RFC(\%) \\
\cline { 2 - 7 } Vf & 0,006 & 0,007 & 0,0008 & 2,55 & 0,16 & 0,22 & 14,14 \\
Ve & 0,002 & 0,001 & 0,0003 & 0,60 & 0,10 & 0,10 & 3,48 \\
Vg & 0,004 & 0,006 & 0,0005 & 1,95 & 0,06 & 0,12 & 10,66 \\
CVf (\%) & 5,30 & 6,96 & 9,90 & 6,03 & 11,33 & 15,95 & 5,73 \\
CVe (\%) & 3,06 & 3,14 & 6,38 & 2,93 & 8,96 & 10,76 & 2,84 \\
CVg (\%) & 4,33 & 6,21 & 7,58 & 5,27 & 6,94 & 11,78 & 4,97 \\
CVr & 1,42 & 1,98 & 1,19 & 1,80 & 0,77 & 1,09 & 1,75 \\
MÉDIA & 1,46 & 1,17 & 0,29 & 26,48 & 3,53 & 2,94 & 65,63 \\
\hline
\end{tabular}

PF: peso do fruto; PS: peso da semente; PP: peso da polpa; RPF: rendimento de polpa por fruto; PC: peso do cacho; PFC: peso de frutos por cacho; RFC: rendimento de fruto por cacho; Vf, Ve, Vg, CVf, CVe, CVg, CVr e CVg/CVe: vide Tabela 3.

Fonte: Elaboração dos autores.

Tabela 5. Estimativas de parâmetros genéticos obtidos da análise de 129 matrizes de açaizeiro coletadas em seis municípios do Nordeste Paraense em relação aos caracteres NRC, NFR, CC, CIC, PCF, NCP, NFC e PTF. Belém-PA, UFRA, 2012.

\begin{tabular}{lrrrrrrrr}
\hline \multirow{1}{*}{ Parâmetros } & NRC & NFR & CC(cm) & CIC(cm) & PCF(g) & NCP & NFC & PTF(kg) \\
\cline { 2 - 8 } Vf & 0,14 & 0,17 & 18,69 & 43,57 & 70,41 & 0,022 & 22,31 & 9,91 \\
Ve & 0,05 & 0,05 & 14,48 & 29,11 & 23,26 & 0,017 & 5,81 & 3,13 \\
Vg & 0,09 & 0,12 & 4,21 & 14,46 & 47,15 & 0,005 & 16,50 & 6,78 \\
CVf (\%) & 3,82 & 8,98 & 5,46 & 5,63 & 5,63 & 7,53 & 10,49 & 23,98 \\
CVe (\%) & 2,28 & 4,87 & 4,80 & 4,60 & 3,24 & 6,62 & 5,35 & 13,47 \\
CVg (\%) & 3,06 & 7,55 & 2,59 & 3,24 & 4,61 & 3,59 & 9,02 & 19,83 \\
CVr & 1,34 & 1,55 & 0,54 & 0,70 & 1,42 & 0,54 & 1,69 & 1,47 \\
MÉDIA & 9,80 & 4,59 & 79,21 & 117,33 & 149,04 & 1,97 & 45,03 & 13,13 \\
\hline
\end{tabular}

NRC: número de ráquilas por cacho; NFR: número de frutos por ráquila; $\mathrm{CC}$ : comprimento do cacho; CIC: circunferência do cacho; PCF: peso de 100 frutos; NCP: número de cachos por planta; NFC: número de frutos por cacho; PTF: produção total dos frutos; Vf, Ve, Vg, CVf, CVe, CVg, CVr e CVg/CVe: vide Tabela 3.

Fonte: Elaboração dos autores. 
A variação nas estimativas de dissimilaridade fenotípica entre as 8.256 combinações referente as 129 matrizes de açaizeiro, avaliadas com base em 14 caracteres previamente selecionados por ACP (GALATE et al., 2012) foi bastante elevada, os valores observados entre os pares variaram de 0,466 a 3,149, indicando ampla variabilidade genética no conjunto, com amplitude maior que a encontrada por Oliveira, Ferreira e Santos (2007) em estudo sobre divergência genética de acessos de açaizeiro fundamentada em descritores morfoagronômicos.

A Tabela 6 apresenta apenas 20 das combinações menos divergentes e as respectivas estimativas de dissimilaridades $\left(\mathrm{d}_{\mathrm{ij}}\right)$. A maior similaridade genética foi verificada entre as matrizes E0-070 e E0-072 $\left(d_{i j}=0,446\right)$, seguida das combinações EO-064 x EO-071 $\left(\mathrm{d}_{\mathrm{ij}}=0,504\right)$ e EO-106 x EO$117\left(\mathrm{~d}_{\mathrm{ij}}=0,507\right)$, todas da Ilha do Combu. A menor variabilidade entre esses pares de matrizes pode ser explicada devido à coleta ter sido realizada em localidades muito próximas geograficamente, em propriedades de ribeirinhos do rio Guamá, o que possibilita o surgimento de cruzamentos entre indivíduos aparentados ou de autofecundações (SOUZA, 2002). As matrizes EO-012 (São João de Pirabas), EO-064, EO-067 e EO-072 (as três da Ilha do Combu) ocorreram em diversas combinações, o que indica alta similaridade genética em relação às outras matrizes.

Tabela 6. Síntese da matriz de distância euclidiana média entre as 129 matrizes de açaizeiro mais similares, avaliadas em 14 caracteres morfoagronômicos. Belém PA, UFRA, 2012.

\begin{tabular}{|c|c|c|c|c|c|}
\hline \multicolumn{5}{|c|}{ Combinação } & \multirow{2}{*}{$\frac{\text { Distânci }}{\mathbf{d}_{\mathrm{ij}}}$} \\
\hline Matriz1 & LOCAL & & Matriz2 & Local & \\
\hline EO-070 & Ilha do Combu & $\mathrm{x}$ & EO-072 & Ilha do Combu & 0,466 \\
\hline EO-064 & Ilha do Combu & $\mathrm{x}$ & EO-071 & Ilha do Combu & 0,504 \\
\hline EO-106 & Ilha do Combu & $\mathrm{x}$ & EO-117 & Ilha do Combu & 0,507 \\
\hline EO-107 & Ilha do Combu & $\mathrm{x}$ & EO-028 & Santarém Novo & 0,512 \\
\hline EO-015 & Salinópolis & $\mathrm{x}$ & EO-020 & Salinópolis & 0,519 \\
\hline EO-096 & Ilha do Combu & $\mathrm{x}$ & EO-120 & Ilha do Combu & 0,522 \\
\hline EO-088 & Ilha do Combu & $\mathrm{x}$ & EO-126 & Ilha do Combu & 0,542 \\
\hline EO-066 & Ilha do Combu & $\mathrm{x}$ & EO-081 & Ilha do Combu & 0,552 \\
\hline EO-031 & São João de Pirabas & $\mathrm{x}$ & EO-059 & Ilha do Combu & 0,579 \\
\hline EO-034 & Capitão Poço & $\mathrm{x}$ & EO-037 & Capitão Poço & 0,594 \\
\hline EO-064 & Ilha do Combu & $\mathrm{x}$ & EO-067 & Ilha do Combu & 0,599 \\
\hline EO-012 & São João de Pirabas & $\mathrm{x}$ & EO-025 & Santarém Novo & 0,616 \\
\hline EO-023 & Santarém Novo & $\mathrm{x}$ & EO-067 & Ilha do Combu & 0,616 \\
\hline EO-055 & Ilha do Combu & $\mathrm{x}$ & EO-057 & Ilha do Combu & 0,622 \\
\hline EO-080 & Ilha do Combu & $\mathrm{x}$ & EO-097 & Ilha do Combu & 0,623 \\
\hline EO-045 & Capitão Poço & $\mathrm{x}$ & EO-047 & Capitão Poço & 0,635 \\
\hline EO-041 & Ourém & $\mathrm{x}$ & EO-072 & Ilha do Combu & 0,638 \\
\hline EO-061 & Ilha do Combu & $\mathrm{x}$ & EO-064 & Ilha do Combu & 0,640 \\
\hline EO-006 & Ilha do Mosqueiro & $\mathrm{x}$ & EO-012 & São João de Pirabas & 0,640 \\
\hline EO-012 & São João de Pirabas & $\mathrm{x}$ & EO-021 & Salinópolis & 0,643 \\
\hline
\end{tabular}

Fonte: Elaboração dos autores.

Na Tabela 7, pode-se observar as 20 combinações mais divergentes e suas respectivas estimativas de dissimilaridades. Nota-se que a maior dissimilaridade ocorreu entre as matrizes EO-010 (Belém) x EO-018
(Salinópolis), $\mathrm{d}_{\mathrm{ij}}=3,149$; seguidas das combinações entre as matrizes EO-062 (Ilha do Combu) x EO-010 e EO-010 x EO-051 (Capitão Poço), cujas distâncias foram 2,994 e 2,896, respectivamente. As matrizes 
EO-010 e EO-062 foram as que apresentaram maior diversidade genética, uma vez que ocorreram em várias das combinações mais divergentes. Estas matrizes podem ser consideradas uma das melhores opções de genitores em programas de melhoramento genético. Maior destaque deve ser dado à matriz EO010, que apareceu 19 vezes, das 20 combinações mais dissimilares.

As combinações entre as matrizes referenciadas na Tabela 7 são todas promissoras para serem usadas em cruzamentos para obtenção de genótipos superiores, como exemplo, pode-se mencionar a combinação entre as matrizes EO-010 x EO018, de maior dissimilaridade, podendo-se obter descendentes com maior variabilidade genética e maior efeito heterótico.
Oliveira, Ferreira e Santos (2007), no estudo de germoplasma de açaizeiro procedentes dos Estados do Maranhão, Amapá e Pará as distâncias euclidianas entre os pares de acessos variaram de 0,09 a 1,87, com média de 1,39 , sendo que a menor distância foi observada nos acessos procedentes de municípios da Ilha do Marajó (Chaves e Breves), enquanto que as maiores distâncias ocorreram em Breves (PA) e Oiapoque (AP). Farias Neto e Oliveira (2012), as estudar a divergência genética em 20 acessos de tucumanzeiro (Astrocaryum vulgare Mart.), com oito caracteres observou valores mínimo de 0,2433 e máximo de 2,9627. Este valor máximo é especialmente muito próximo ao observado em açaizeiro.

Tabela 7. Síntese da matriz de distância euclidiana média entre as 129 matrizes de açaizeiro mais dissimilares, avaliadas em 14 caracteres morfoagronômicos. Belém PA, UFRA, 2012.

\begin{tabular}{|c|c|c|c|c|c|}
\hline \multicolumn{5}{|c|}{ Combinação } & \multirow{2}{*}{$\begin{array}{r}\text { Distância } \\
d_{i j}\end{array}$} \\
\hline Matriz1 & Local & & Matriz2 & Local & \\
\hline EO-010 & Belém & $\mathrm{x}$ & EO-018 & Salinópolis & 3,149 \\
\hline EO-010 & Belém & $\mathrm{x}$ & EO-062 & Ilha do Combu & 2,994 \\
\hline EO-010 & Belém & $\mathrm{x}$ & EO-051 & Capitão Poço & 2,896 \\
\hline EO-010 & Belém & $\mathrm{x}$ & EO-060 & Ilha do Combu & 2,892 \\
\hline EO-010 & Belém & $\mathrm{x}$ & EO-056 & Ilha do Combu & 2,888 \\
\hline EO-010 & Belém & $\mathrm{x}$ & EO-011 & São João de Pirabas & 2,874 \\
\hline EO-010 & Belém & $\mathrm{x}$ & EO-016 & Salinópolis & 2,814 \\
\hline EO-010 & Belém & $\mathrm{x}$ & EO-079 & Ilha do Combu & 2,800 \\
\hline EO-010 & Belém & $\mathrm{x}$ & EO-114 & Ilha do Combu & 2,784 \\
\hline EO-007 & Ilha do Mosqueiro & $\mathrm{x}$ & EO-010 & Belém & 2,784 \\
\hline EO-010 & Belém & $\mathrm{x}$ & EO-022 & Santarém Novo & 2,775 \\
\hline EO-010 & Belém & $\mathrm{x}$ & EO-110 & Ilha do Combu & 2,767 \\
\hline EO-010 & Belém & $\mathrm{x}$ & EO-033 & Capitão Poço & 2,757 \\
\hline EO-010 & Belém & $\mathrm{x}$ & EO-013 & Santarém Novo & 2,755 \\
\hline EO-019 & Salinópolis & $\mathrm{x}$ & EO-062 & Ilha do Combu & 2,747 \\
\hline EO-010 & Belém & $\mathrm{x}$ & EO-035 & Capitão Poço & 2,743 \\
\hline EO-010 & Belém & $\mathrm{x}$ & EO-069 & Ilha do Combu & 2,725 \\
\hline EO-010 & Belém & $\mathrm{x}$ & EO-023 & Santarém Novo & 2,703 \\
\hline EO-004 & Ilha do Mosqueiro & $\mathrm{x}$ & EO-010 & Belém & 2,699 \\
\hline EO-010 & Belém & $\mathrm{x}$ & EO-055 & Ilha do Combu & 2,686 \\
\hline
\end{tabular}

Fonte: Elaboração dos autores. 
$\mathrm{Na}$ análise de agrupamento pelo método de otimização Tocher, com base nas estimativas da distância euclidiana média das 129 matrizes, foi verificada a formação de 10 grupos distintos. O primeiro grupo foi o mais numeroso, constituído de 107 matrizes, equivalente a 82,9\%, procedentes da Ilha do Combu (68), Capitão Poço (15), Santarém Novo (7), São João de Pirabas (6), Ilha de Mosqueiro (6), Salinópolis (3), Belém (2) e Ourém (1) (Tabela 8).
No grupo II, formado de quatro matrizes, distribuídas nas localidades de Capitão Poço (2), Ilha do Combu (1) e Ilha do Mosqueiro (1).

No grupo III, formado de cinco matrizes provenientes da Ilha do Combu (2), Ourém (2) e Belém (1).

No grupo IV, formado de quatro matrizes procedentes da Ilha do Combu.

No grupo $\mathrm{V}$, formado de três matrizes oriundas de Salinópolis (2) e São João de Pirabas (1).

Tabela 8. Grupos de similaridade genética entre 129 matrizes de açaizeiro, mediante a utilização de 14 caracteres selecionados, gerados pelo método de otimização de Tocher com base na distância euclidiana média. Belém-PA, UFRA, 2012.

\begin{tabular}{|c|c|c|c|c|c|c|c|c|c|c|c|c|}
\hline \multirow[t]{2}{*}{ Grupo } & \multicolumn{12}{|c|}{ Matriz* } \\
\hline & 70 & 72 & 41 & 71 & 64 & 12 & 25 & 39 & 102 & 57 & 67 & 21 \\
\hline & 37 & 65 & 2 & 15 & 31 & 127 & 34 & 68 & 55 & 87 & 28 & 108 \\
\hline & 6 & 47 & 107 & 59 & 96 & 99 & 78 & 75 & 121 & 61 & 48 & 1 \\
\hline & 77 & 89 & 24 & 20 & 66 & 92 & 85 & 13 & 23 & 93 & 126 & 88 \\
\hline I & 50 & 73 & 81 & 103 & 74 & 86 & 120 & 82 & 104 & 98 & 116 & 113 \\
\hline & 106 & 32 & 129 & 63 & 84 & 27 & 117 & 90 & 5 & 26 & 52 & 45 \\
\hline & 3 & 58 & 40 & 4 & 105 & 9 & 38 & 91 & 119 & 112 & 125 & 123 \\
\hline & 122 & 95 & 100 & 83 & 115 & 97 & 44 & 118 & 46 & 94 & 76 & 80 \\
\hline & 69 & 128 & 124 & 51 & 114 & 22 & 29 & 30 & 36 & 53 & 110 & \\
\hline II & 7 & 49 & 60 & 33 & & & & & & & & \\
\hline III & 8 & 43 & 54 & 42 & 101 & & & & & & & \\
\hline IV & 56 & 62 & 79 & 111 & & & & & & & & \\
\hline $\mathbf{V}$ & 14 & 17 & 16 & & & & & & & & & \\
\hline VI & 11 & 18 & & & & & & & & & & \\
\hline VII & 35 & & & & & & & & & & & \\
\hline VIII & 109 & & & & & & & & & & & \\
\hline IX & 19 & & & & & & & & & & & \\
\hline $\mathbf{X}$ & 10 & & & & & & & & & & & \\
\hline
\end{tabular}

*/Na Tabela constam apenas os números de entrada das matrizes, para simplificar sua apresentação.

Fonte: Elaboração dos autores.

No grupo VI, formado de duas matrizes procedentes de Salinópolis (1) e São João de Pirabas (1).

Os grupos VII, VIII, IX e X foram constituídos pelas matrizes EO-035 (Capitão Poço), EO-109 (Ilha do Combu), EO-019 (Salinópolis) e EO010 (Belém), distribuídas em grupos isolados e, possivelmente, apresentam maior dissimilaridade média em relação às demais avaliadas. 
Oliveira, Ferreira e Santos (2007), em estudo sobre divergência genética entre 87 acessos de açaizeiro com base em 22 caracteres morfoagronômicos previamente selecionados por análise de componentes principais (ACP) procedentes do Maranhão, do Amapá e do Pará, com apenas uma procedência em comum com o atual estudo (Ilha do Mosqueiro), verificaram a formação de 24 agrupamentos pelo método de Tocher, os quais foram constituídos por poucos acessos, de dois a quatro, com exceção do último grupo, formado por apenas um acesso.

Por outro lado, Manfio (2010), usando o mesmo método em análise genética para o melhoramento da macaúba, com base em caracteres não selecionados por ACP, entre 145 matrizes procedentes dos Estados de Minas Gerais (91), Mato Grosso do Sul (41), São Paulo (10), Pernambuco (1), Pará (1) e Maranhão (1) verificou a formação de nove grupos distintos com maior concentração de matrizes nos dois primeiros grupos, e os três últimos grupos com apenas uma matriz. Quando analisou somente as matrizes de Minas Gerais, observou a formação de 17 grupos, com maior densidade no grupo I, 25 matrizes; e os últimos sete grupos, com apenas uma matriz cada.

A matriz EO-010 (Belém), isolada no último grupo, na análise intergrupos, foi a mais divergente em relação a todo conjunto genotípico, uma vez que esse grupo apresentou a maior divergência com os demais. Porém, as matrizes EO-011 (São João de Pirabas), EO-018 e EO-019 (Salinópolis), EO-035 (Capitão Poço), EO-062 e EO-109 (Ilha do Combu) também apresentam dissimilaridade genética, constituindo-se em boas opções de parentais, por isso podem ser indicadas para um programa de melhoramento genético, desde que os cruzamentos apresentem características agronômicas desejáveis, visando obter a melhoria de caracteres quantitativos.
No dendrograma estabelecido com base no método UPGMA para identificar os grupos das 129 matrizes, avaliadas em 14 caracteres morfoagronômicos selecionados por ACP (GALATE et al., 2012), foi observada a formação de nove grupos, utilizando $65 \%$ de dissimilaridade como critério de definição (Figura 1). Os grupos de similaridades constam na Tabela 9.

O grupo I foi formado de 104 matrizes, equivalente a $81 \%$, sendo da Ilha do Combu (68), Capitão Poço (13), Santarém Novo (6), São João de Pirabas (6), Ilha de Mosqueiro (4), Salinópolis (3), Ourém (2) e Belém (2). O grupo II foi formado de cinco matrizes, sendo de Capitão Poço (2), Ilha do Combu (1), Belém (1) e Ourém (1). O grupo III foi formado de 11 matrizes, sendo de Capitão Poço (3), Ilha do Combu (3), Ilha do Mosqueiro (2), São João de Pirabas (1), Salinópolis (1) e Santarém Novo (1). O grupo IV foi formado de três matrizes, todas da Ilha do Combu. O grupo $\mathrm{V}$ foi formado apenas de uma matriz da Ilha do Combu. O grupo VI foi formado por duas matrizes, sendo de São João de Pirabas (1) e Salinópolis (1). Os grupos VII e VIII apresentaram duas matrizes procedentes de Salinópolis e, o grupo IX, apenas de uma matriz procedente de Belém.

Este método isolou quatro matrizes, correspondentes aos grupos V, VII, VIII e IX, definidos como dissimilares em relação aos demais, consequentemente, com matrizes mais divergentes, verificando-se que a matriz EO-010 (Belém) foi classificada como a mais divergente entre todas as matrizes.

Além da matriz EO-010 (Belém), as matrizes EO-011 (São João de Pirabas), EO-017, EO018 e EO-019 (Salinópolis); EO-062 e EO-109 (Ilha do Combu) foram as mais promissoras, com viabilidade de serem utilizadas em programas de melhoramento genético de açaizeiro para caracteres morfoagronômicos quantitativos. 
Figura 1. Agrupamento das 129 matrizes de açaizeiro procedentes de seis municípios do Nordeste Paraense pelo método de UPGMA, baseado na divergência genética expressa pela distância euclidiana média. Belém-PA, UFRA, 2012.

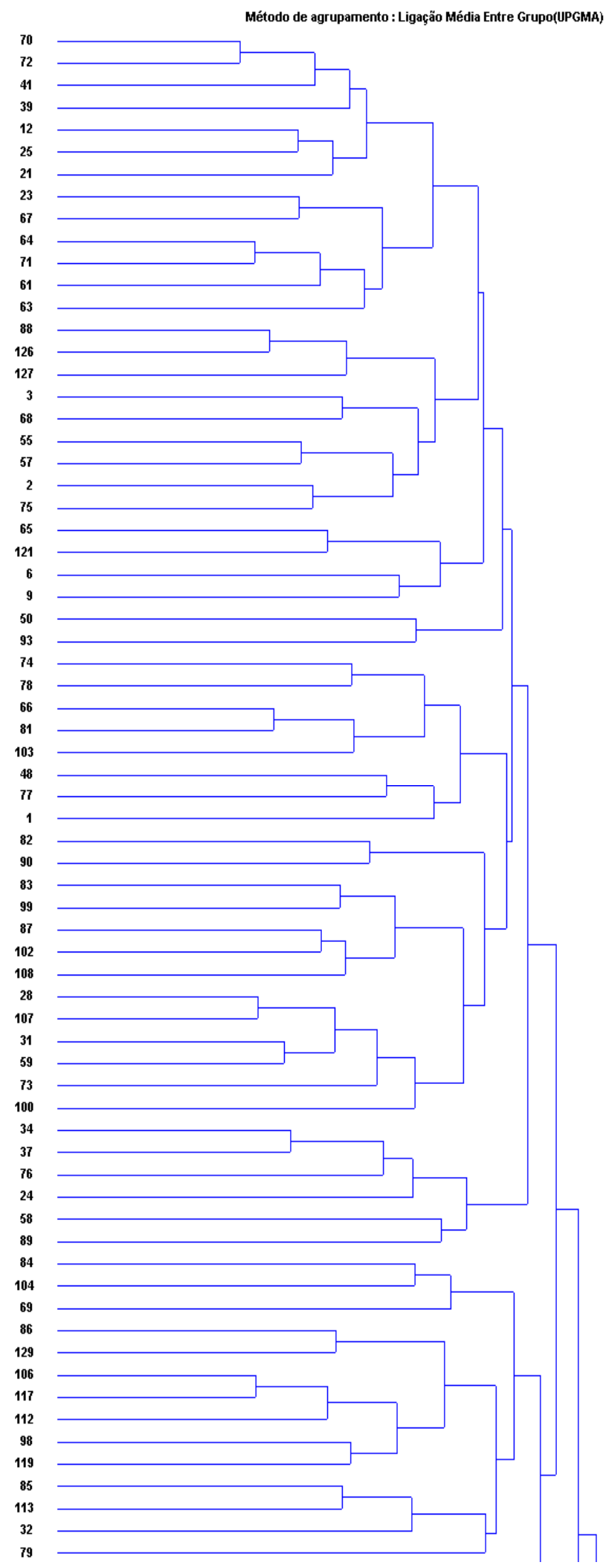

continua 
Galate, R. S. et al.

continuação

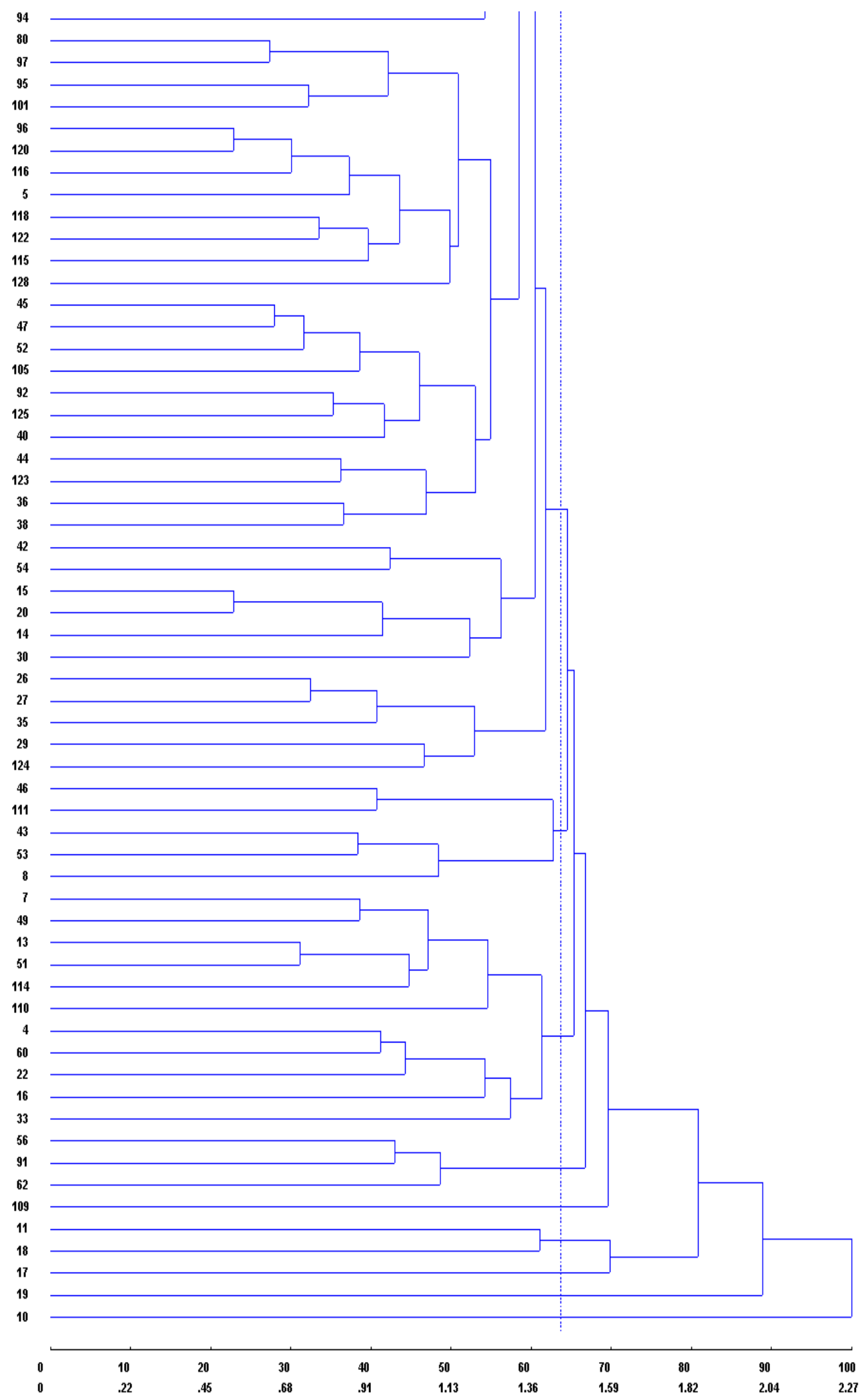

Fonte: Elaboração dos autores. 
Tabela 9. Grupos de similaridade genética entre 129 matrizes de açaizeiro, mediante a utilização de 14 caracteres selecionados, estabelecidos pelo método de UPGMA, com base na distância euclidiana média. Belém-PA, UFRA, 2012.

\begin{tabular}{|c|c|c|c|c|c|c|c|c|c|c|c|c|c|}
\hline \multirow{2}{*}{$\begin{array}{c}\text { Grupos } \\
\end{array}$} & \multicolumn{13}{|c|}{ Matrizes* } \\
\hline & 1 & 2 & 3 & 5 & 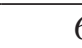 & 6 & 9 & 12 & 14 & 15 & 20 & 21 & 23 \\
\hline & 24 & 25 & 26 & 27 & $2 \varepsilon$ & & 29 & 30 & 31 & 32 & 34 & 35 & 36 \\
\hline & 37 & 38 & 39 & 40 & 41 & & 42 & 44 & 45 & 47 & 48 & 50 & 52 \\
\hline & 54 & 55 & 57 & 58 & 5 & & 61 & 63 & 64 & 65 & 66 & 67 & 68 \\
\hline & 69 & 70 & 71 & 72 & 73 & & 74 & 75 & 76 & 77 & 78 & 79 & 80 \\
\hline I & 81 & 82 & 83 & 84 & 85 & & 86 & 87 & 88 & 89 & 90 & 92 & 93 \\
\hline & 94 & 95 & 96 & 97 & 98 & & 99 & 100 & 101 & 102 & 103 & 104 & 105 \\
\hline & 106 & 107 & 108 & 112 & 113 & & 115 & 116 & 117 & 118 & 119 & 120 & 121 \\
\hline & 122 & 123 & 124 & 125 & 126 & & 127 & 128 & 129 & & & & \\
\hline II & 8 & 43 & 46 & 53 & 111 & & & & & & & & \\
\hline III & 4 & 7 & 13 & 16 & & 233 & & 49 & 51 & 60 & 110 & 114 & \\
\hline IV & 56 & 62 & 91 & & & & & & & & & & \\
\hline $\mathbf{V}$ & 109 & & & & & & & & & & & & \\
\hline VI & 11 & 18 & & & & & & & & & & & \\
\hline VII & 17 & & & & & & & & & & & & \\
\hline VIII & 19 & & & & & & & & & & & & \\
\hline IX & 10 & & & & & & & & & & & & \\
\hline
\end{tabular}

*/Na Tabela constam apenas os números de entrada das matrizes, para simplificar sua apresentação.

Fonte: Elaboração dos autores.

Vieira et al. (2013), ao estudarem a variabilidade fenotípica de 16 acessos de mandioca da Embrapa Cerrados por meio de caracteres fenotípicos quantitativos, qualitativos e caracteres moleculares verificaram haver ampla variabilidade nos caracteres examinados, havendo baixa correlação entre as medidas de dissimilaridade utilizadas, o que indicou que o estudo da divergência genética. é o mais recomendado para orientar decisões sobre conservação e utilização de germoplasma.

Oliveira,Ferreirae Santos(2007), em estudo sobre divergência genética entre acessos de açaizeiro com base em caracteres morfoagronômicos selecionados por ACP, verificaram a formação de cinco grupos pelo método de UPGMA; os dois primeiros foram formados por 11 acessos, considerados os mais divergentes, sendo que o último grupo reuniu 33 acessos, provavelmente os menos divergentes, não houve nenhum grupo isolado.

Em estudo sobre diversidade genética de macaúba, Manfio (2010), empregando o método de UPGMA com base em caracteres originais, não selecionados por $\mathrm{ACP}$, detectou cinco grupos formados por matrizes de mais de uma procedência, sem tendência em relação à origem geográfica, não havendo isolamento de nenhuma matriz. Porém, ao analisar 91 matrizes de um mesmo Estado Federativo (MG), observou a formação de oito 
grupos, com os dois primeiros reunindo 30 e 32 matrizes, respectivamente e, nos grupos VI e VII, detectou matrizes isoladas.

Pelos dois métodos, Tocher e UPGMA, utilizados nos trabalhos desses autores observou-se variabilidade nos acessos provenientes de mesmos municípios, ao contrário do que foi observado neste estudo, que apresentou pouca variabilidade em matrizes da mesma localidade, como no caso da maioria das matrizes da Ilha do Combu, concentradas no grupo I.

O número de grupos formados pelos dois métodos foi muito semelhante e apresentaram ampla variabilidade entre os genótipos estudados. Para o melhoramento genético, é importante a separação das matrizes em grupos distintos, uma vez que, quando se realizar cruzamentos entre indivíduos de grupos diferentes pode-se obter heterose.

Os dois métodos alocaram as 129 matrizes em grupos muito semelhantes, sendo que o método de Tocher apresentou a distância média dentro dos grupos sempre menor que a distância média entre grupos. $\mathrm{O}$ que indica que as matrizes de um mesmo grupo são mais similares do que as matrizes de grupos diferentes. No estudo da divergência genética, a utilização conjunta do método de otimização de Tocher com o método de UPGMA é recomendada.

Concluiu-se, com base nos resultados obtidos, que $77,3 \%$ dos 22 caracteres morfoagronômicos avaliados fornecem informações de que as 129 matrizes de açaizeiro do Nordeste Paraense apresentam elevada variabilidade fenotípica. $\mathrm{Na}$ avaliação preliminar dos parâmetros genéticos, $40,9 \%$ dos caracteres examinados tiveram componente de variância genética acima de 0,9 e $72,7 \%$ apresentando razão $\mathrm{CVg} / \mathrm{CVe}$ superior a um, indicando ampla variabilidade genética na coleção de germoplasma estudada. $\quad \mathrm{O}$ estudo da distância euclidiana e análise de agrupamento pelos métodos de Tocher e UPGMA identificaram matrizes divergentes para utilização como genitores na obtenção de genótipos superiores, com destaque para as matrizes EO-010 (Belém) e EO-062 (Ilha do Combu); EO-010 (Belém); EO-011 (São João de Pirabas), EO-017, EO-018 e EO-019 (Salinópolis); EO-062 e EO-109 (Ilha do Combu).

\section{Referências}

CRUZ, C. D. Programa genes: análise multivariada e simulação. Viçosa, MG: UFV, 2006. v. 1. 17 5p.

Programa genes: diversidade genética. ViçosaMG: UFV, 2008. v. 1.278 p.

FALCONER, D. S.; MACKAY, T. F. Introduction to quantitative genetics. 4. ed. Londres: Longman Group, 1996. $464 \mathrm{p}$.

FARIAS NETO, J. T.; OLIVEIRA, M. S. P. Divergência genética entre acessos de tucumanzeiro (Astrocaryum vulgare Mart.) para caracteres do fruto. Belém: Embrapa-Cpatu, 2012. Disponível em: <http://www. alice.cnptia.embrapa.br/bitstream/doc/406671/1/038. pdf $>$. Acesso em: 15 mar. 2012.

FARIAS NETO, J. T.; RESENDE, M. D. V.; OLIVEIRA, M. S. P.; SANTOS, N. S. A.; CANUTO, E. L.; NOGUEIRA, L. O.; MULLER, A. A. Avaliação genética de progênies de polinização aberta de açaí (Euterpe oleracea) e estimativas de parâmetros genéticos. Revista Cerne, Lavras, v. 13, n. 4, p. 376383, out./dez. 2007.

GALATE, R. S.; MOTA, M. G. C.; GAIA, J. M. D.; COSTA, M. S. S. Caracterização morfoagronômica de germoplasma de açaizeiro no Nordeste Paraense. Revista Brasileira de Fruticultura, Jaboticabal, v. 34, n. 2, p. 540-550, 2012.

HOMMA,A. K. O. Sistema de produção do açaí-mercado e comercialização. 2. ed. Belém: Embrapa Amazônia Oriental, 2006. (Sistemas de Produção, 4). Disponível em: <http://sistem asdeproducao.cnptia. embrapa.br/ FontesHTML/Acai/SistemaProducaoAcai_2ed/index. $\mathrm{htm}>$. Acesso em: 5 maio 2011.

MANFIO, C. E. Análise genética no melhoramento da macaúba. 2010. Tese (Doutorado em Genética e Melhoramento) - Curso de Pós Graduação em Genética e Melhoramento. Universidade Federal de Viçosa, Viçosa.

OLIVEIRA, M._S. P.; FERREIRA, D. F.; SANTOS, J. $B$. Divergência genética de açaizeiro fundamentada em descritores morfoagronômicos. Pesquisa Agropecuária Brasileira, Brasília, v. 42, n. 4, p. 501-506, abr. 2007. 
Seleção de descritores para caracterização de germoplasma de açaizeiro para produção de frutos. Pesquisa Agropecuária Brasileira, Brasília, v. 41, n. 7, p. 1133-1140, jul. 2006.

SANTANA, A. C.; CARVALHO, D. F.; MENDES, F. A. T. Análise sistêmica da fruticultura paraense: organização, mercado e competitividade empresarial. Belém: Banco da Amazônia, 2008. 255 p.

SOUZA, P. C. A. Aspectos ecológicos e genéticos de uma população natural de Euterpe oleracea Mart. no Estuário Amazônico. Piracicaba, 2002. Dissertação (Mestrado em Recursos Florestais) - Curso de Pós-Graduação em Recursos Florestais. Escola Superior de Agricultura Luiz de Queiroz. Universidade de São Paulo, São Paulo.

VENCOVSKY, R.; BARRIGA, P. Genética biométrica no fitomelhoramento. Ribeirão Preto: SBG, 1992. 496 p. VIEIRA, E. A.; CARVALHO, F. I. F.; OLIVEIRA, A. C.;
MARTINS, L. F.; BENIN, G.; SILVA, J. A. G.; KOPP, M. M.; HARTWIG, I.; CARVALHO, M. F.; VALÉRIO, I. P. Associação da distância genética em trigo estimada a partir de caracteres morfológicos, caracteres fenológicos e dos componentes do rendimento de grãos. Revista Brasileira de Agrociência, Pelotas, v. 13, n. 2, p. 161168, abr./jun. 2007.

VIEIRA, E. A.; FIALHO, J. F.; FALEIRO, F. G.; BELLON, G.; FONSECA, K. G.; SILVA, M. S.; PAULA-MORAES, S. V.; CARVALHO, L. J. C. B. Semina: Ciências Agrárias, Londrina, v. 34, n. 2, p. $567-$ 582, mar./abr. 2013.

YOKOMIZO, G. K. I.; QUEIROZ, J. A. L.; MOCHIUTTI, S.; PINHEIRO, I. N.; SILVA, P. A. R. Desempenho de progênies de açaizeiros avaliadas para caracteres agronômicos no Estado do Amapá. Scientia Forestalis, Piracicaba, v. 38, n. 87, p. 367-376, set. 2010. 
